\title{
A Finite Element Model of the C1-C4 Human Spine Segment
}

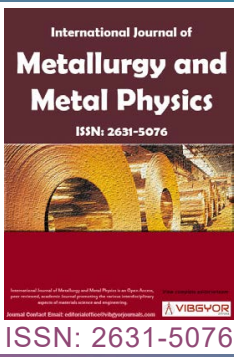

\section{Edison A Bonifaz*}

\section{Mechanical Engineering Department, Universidad San Francisco de Quito, Ecuador}

\begin{abstract}
A three-dimensional FE analysis of four adjacent cervical vertebrae (C1-C4) has been developed. The model is composed of vertebrae and skull but disc ligaments were not considered. The aim of this research is to address the new advancements in the finite element mesh generation of the human cervical spine. The procedure for creating a finite element model based on available biomedical closed volumes obtained from CT scans is introduced. The mesh conversion from triangular to tetrahedral elements, the materialproperty assignment, and the boundary and load conditions were performed with ABAQUS FE code. To assess the overall behavior of the C1-C4 spine segment, the model was subjected to a concentrated load situated in the external occipital protuberance, and every node lying on the inferior surface of the C4 vertebra was encastered. This case study was performed to illustrate the usefulness of the FE model as an important tool in computational spine research as it can provide general information on spinal behavior under various loading conditions.
\end{abstract}

\section{Introduction}

The human spine is a complex structure whose principal functions are to protect the spinal cord and transfer loads from the head and trunk to the pelvis [1]. Advancements in numerical methods during the last decade have enabled researchers to propose more accurate models of the cervical spine. The finite element model has been used as an effective tool in human spine biomechanics. Biomechanical finite element models have provided basic insights into the workings of the cervical spine system. Actual finite element models of the cervical spine consider more accurate representation of the geometry of each vertebra. The generation of 3D FE models of bone from CT scans involves geometry definition and the application of a specific meshing procedure. The complex geometry of the spine is created by combining digitalized quantitative axial computed tomography (CT) scans, and STL-CAD methods. There are numerous commonly open source and proprietary-software programs available for use with DICOM data sets. To generate the FE closed volume geometry of the bone structure, segmentation mask during image processing of medical CT data is currently conducted by using specialist software such as 3D Slicer [2] or Mimics [3], and the specific meshing procedure with software such as OsiriX [4] or MeshLab [5]. 3D Slicer is an open source software platform for medical image informatics, image processing, and three-dimensional visualization. Mesh Lab is the open source system for processing and editing 3D triangular meshes. It provides a set of tools for ed-

*Corresponding author: EA Bonifaz, Mechanical Engineering Department, Universidad San Francisco de Quito, Cumbayá, 170901, Quito, Ecuador, Tel: +593-2-297-1700, ext. 1235

Accepted: February 06, 2021; Published: February 08, 2021

Copyright: (C) 2021 Bonifaz EA. This is an open-access article distributed under the terms of the Creative Commons Attribution License, which permits unrestricted use, distribution, and reproduction in any medium, provided the original author and source are credited.

Bonifaz. Int J Metall Met Phys 2021, 6:063

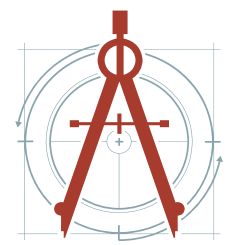


iting, cleaning, healing, inspecting, rendering, texturing and converting meshes. It offers features for processing raw data produced by 3D digitization tools/devices and for preparing .STL models for 3D printing. STL is a file format native to the stereolithography CAD software created by 3D Systems and it is widely used for rapid prototyping, 3D printing and computer-aided manufacturing (Figure 1).

\section{The Model}

In this work, to generate the 3D-FE model, the cervical closed volume it was directly downloaded from the embodi3d web page [6]. The available .STL raw model with $1,035,812$ vertices and $2,071,824$ faces was reduced to 64,644 vertices and 129,488 faces using the code MeshLab [5]. The new .STL file was imported into the FE code ABAQUS [7] for creating an isotropic static finite element model. The entire meshing procedure for the vertebrae was significantly speeded up by using 824503 C3D4 quadratic tetrahedral solid elements.

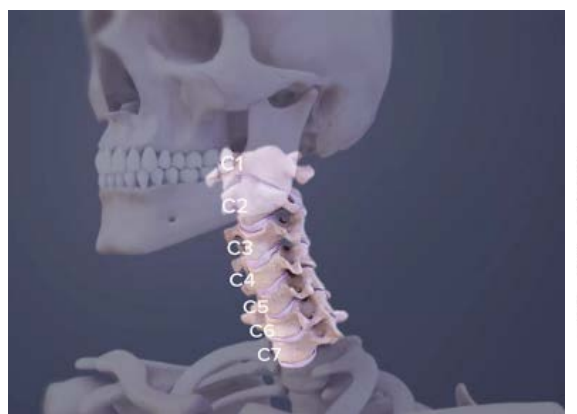

a)

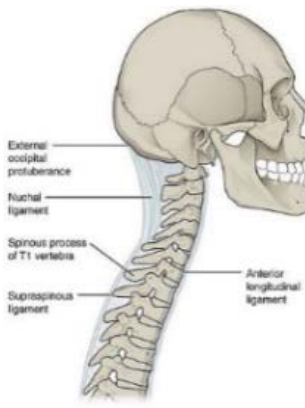

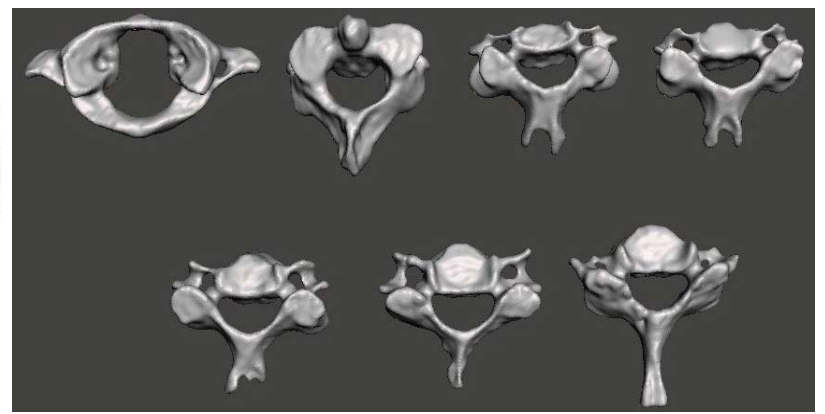

b)

Figure 1: The human cervical spine: a) Raw state of the model with skull C0; b) The 7 stacked bones called vertebrae labeled C1 through C7. The top of the cervical spine connects to the skull, and the bottom connects to the upper back at about shoulder level. As viewed from the side, the cervical spine forms a lordotic curve by gently curving toward the front of the body and then back.
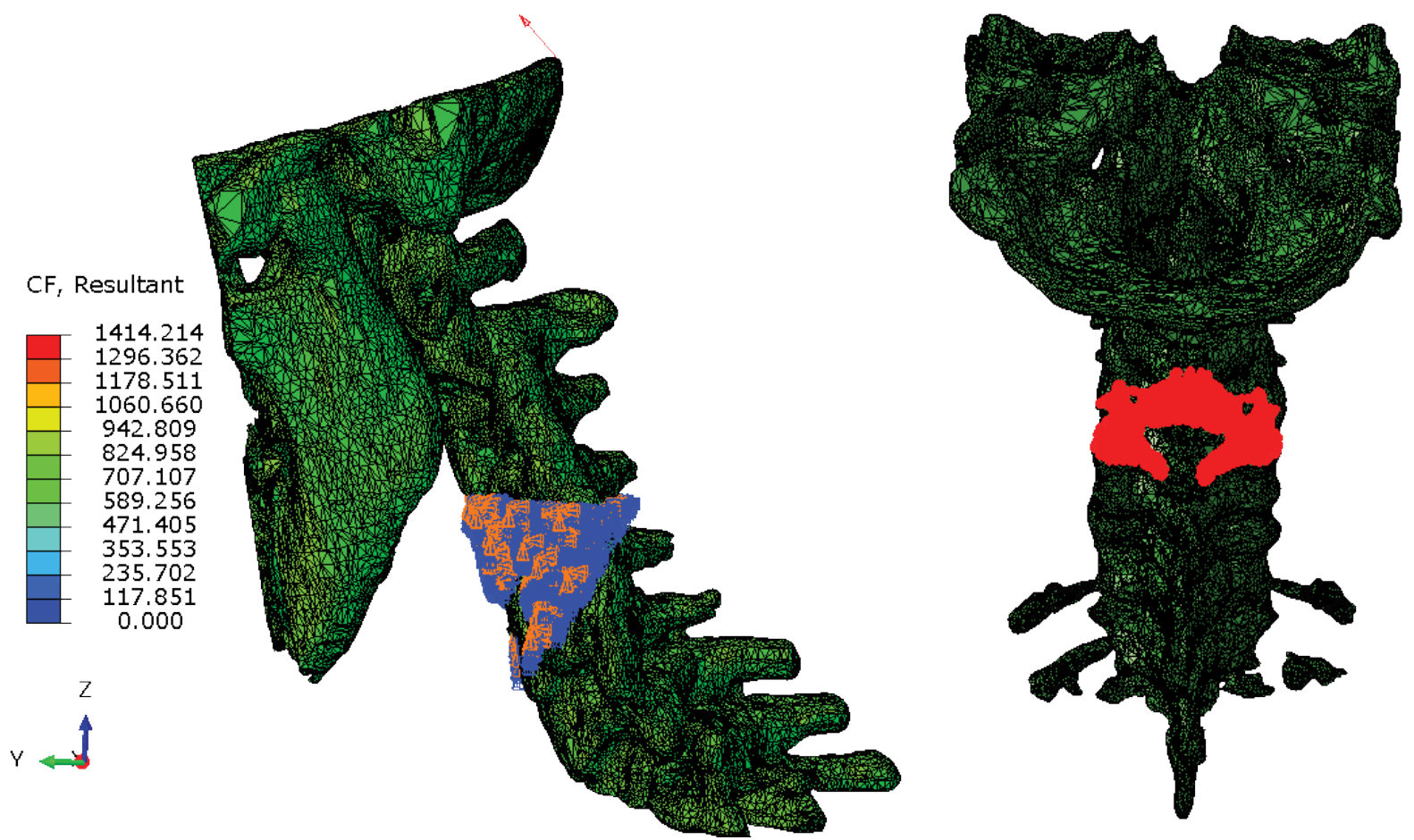

Figure 2: Load and boundary conditions: A concentrated force applied in the external occipital protuberance and encastered BCs applied on the inferior surface of the $\mathrm{C} 4$ vertebra. 


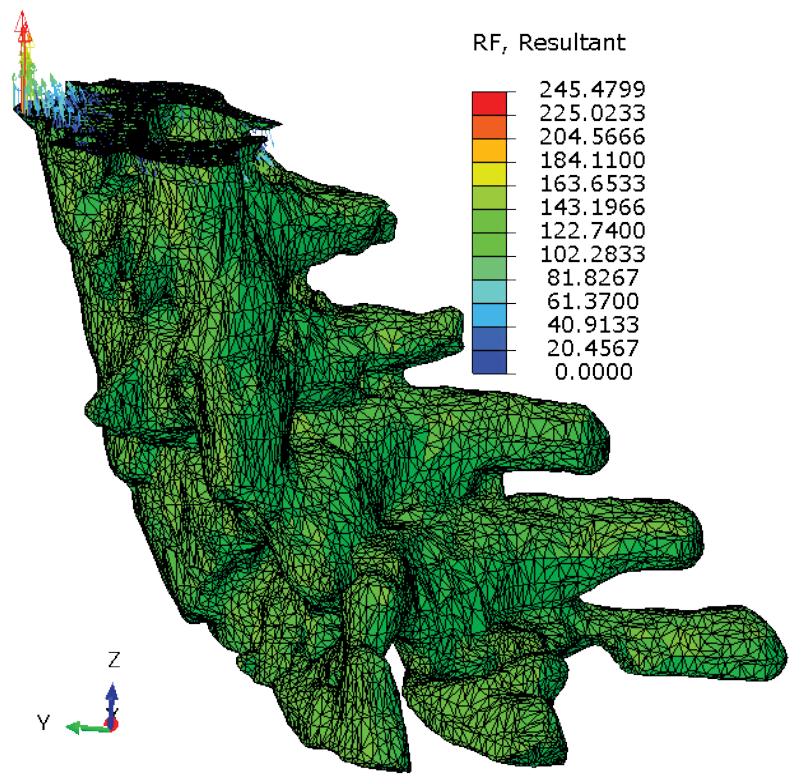

a)

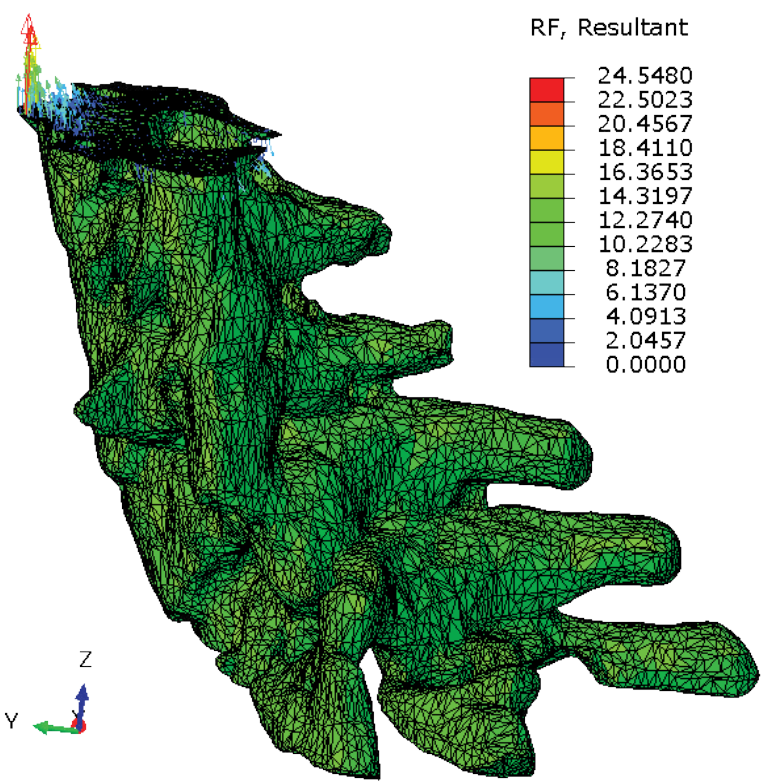

b)

Figure 3: The resultant reaction force distribution on the inferior surface of the C4 vertebra: a) For a concentrated load of $1414.2 \mathrm{~N}$; and b) For a concentrated load of $141.42 \mathrm{~N}$.
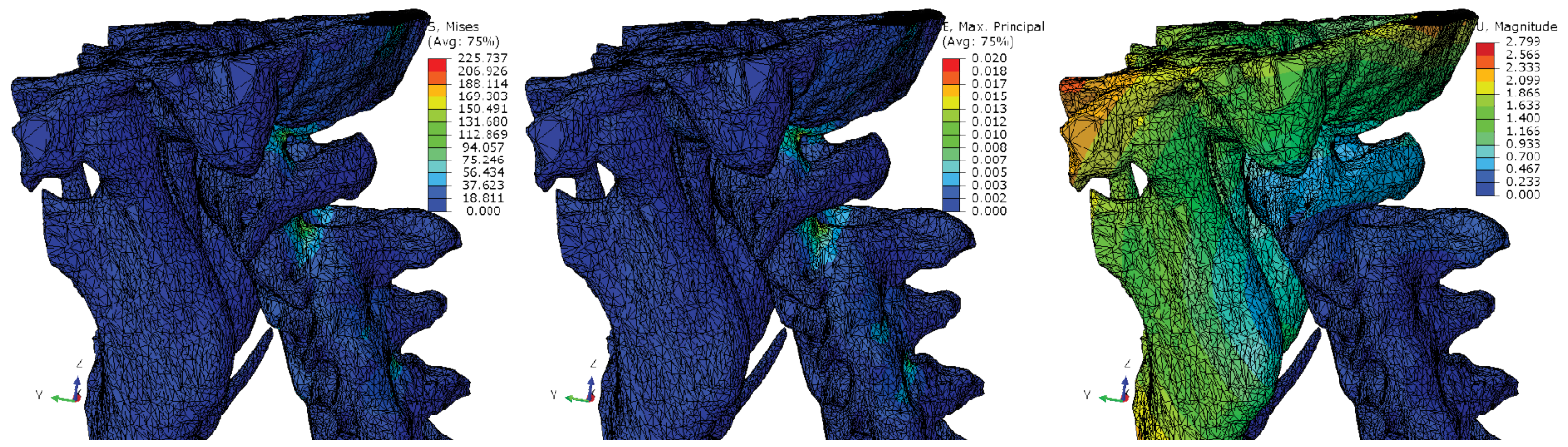

a)
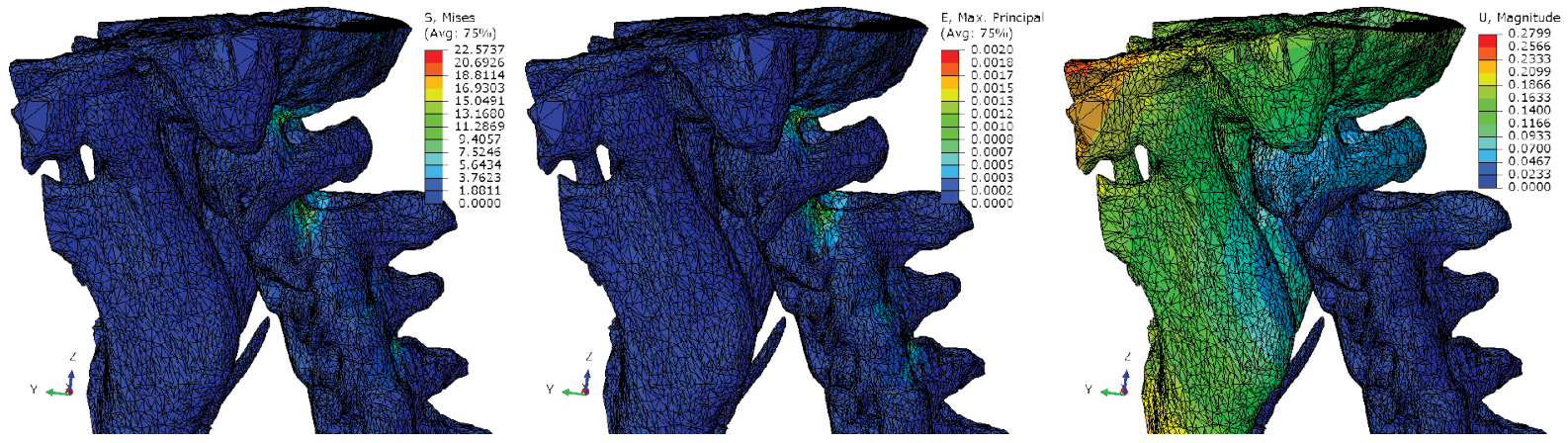

b)

Figure 4: Mises stresses, strains and displacements contours: a) For a concentrated load of $1414.2 \mathrm{~N}$; and b) For a concentrated load of $141.42 \mathrm{~N}$. 


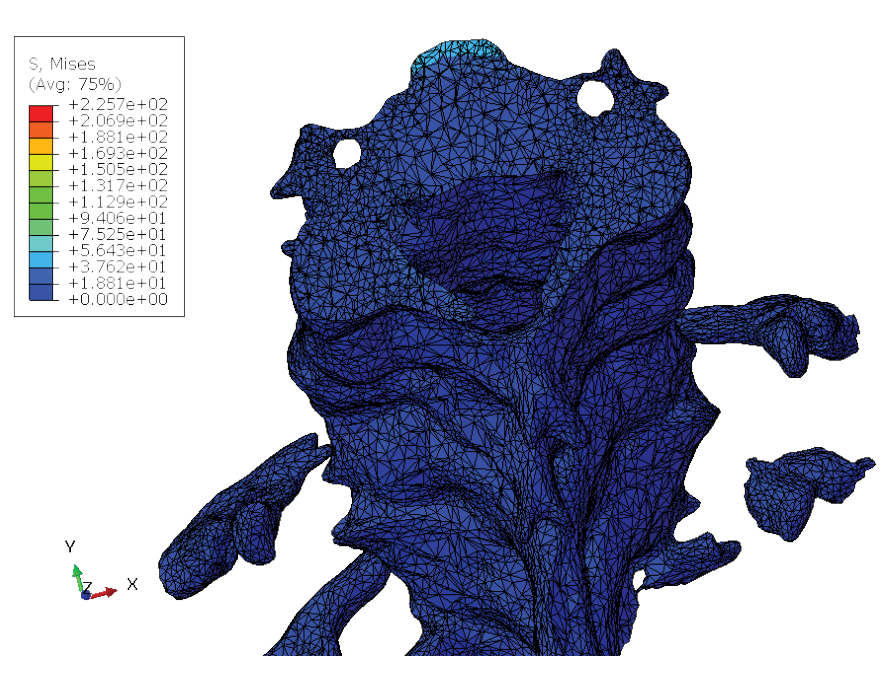

a)

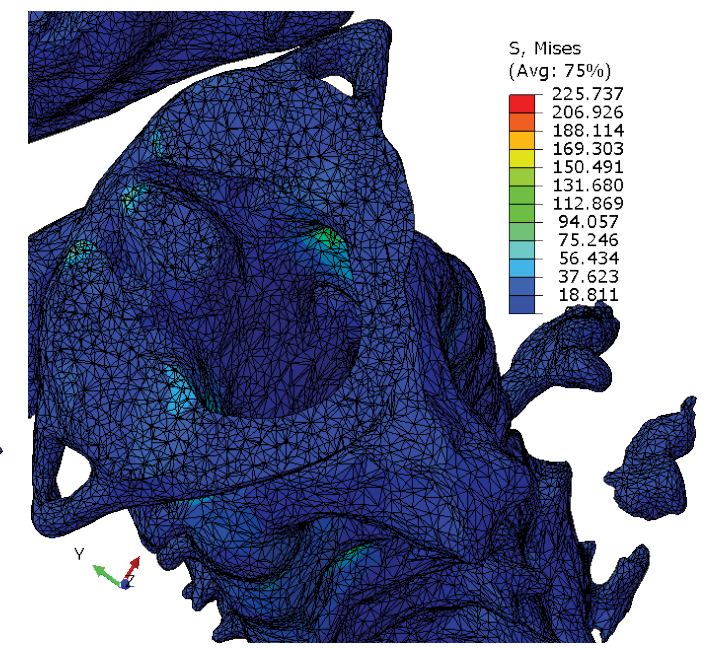

b)

Figure 5: Mises stress contours after the application of a concentrated load of $1414.2 \mathrm{~N}$ : a) On the cross section of a C4 vertebra; b) On the cross section of vertebra $\mathrm{C} 1$.

\section{Boundary and loading condition}

To assess the overall behavior of the C1-C4 spine, the model was subjected to a concentrated load situated in the external occipital protuberance, and to encastered boundary conditions (i.e., zero displacement and rotation) in every node lying on the inferior surface of the $\mathrm{C} 4$ vertebra (Figure 2). Other types of loading, including tension, compression (to represent the weight of the skull), shear, flexion, extension, lateral bending and axial rotation can be applied on the superior aspect of the uppermost vertebra, and a follower load that simulates the body weight may be applied to each vertebra.

\section{Results}

Figure 3, Figure 4 and Figure 5 show ABAQUS finite element results for the configuration shown on Figure 2. A Young's Modulus of $10000 \mathrm{MPa}$ and a poison's ratio of 0.3 were considered in the whole analysis. The plotted Mises stresses and the displacements are in $\mathrm{MPa}$ and in $\mathrm{mm}$ respectively.

It is shown that the deformations do not result in strains that would cause the material to exceed the proportional limit; that is, the material remains within the elastic limits of Hooke's law.

\section{Conclusions}

- It is observed a linear proportionality in the results. That is, for a concentrated load ratio of 10 (1414.2:141.42), the Mises stresses, strains, displacements and reaction forces are in the same 10:1 ratio. This is in correspondence with the isotropic elastic analysis performed to a rigid continuum material that obeys the Hoke's law.

- This case study was performed to illustrate the usefulness of the FE model as an important tool in computational spine research as it can provide general information on spinal behavior under various loading conditions.

\section{References}

1. MA Tyndyk, V Barron, PE Mchugh, DO'mahoney (2007) Generation of a finite element model of the thoracolumbar spine. Acta of Bioengineering and Biomechanics 9: 35-46.

2. https://buildmedia.readthedocs.org/media/pdf/slicer/latest/slicer.pdf

3. https://www.materialise.com/en/medical/mimicsstart-page

4. https://www.osirix-viewer.com/

5. https://www.meshlab.net/

6. https://www.embodi3d.com/

7. https://www.3ds.com/es/productos-y-servicios/ simulia/productos/abaqus/ 